The Open Biomedical Engineering
Journal
Content list available at: $h$ htps://openbiomedicalengineeringjournal.com
fengnecing
loumal

EDITORIAL

\title{
Editorial: Applications of Soft Computing and Machine Learning Techniques for Biomedical Signals and Images
}

\author{
Rahul K. Kher, ${ }^{1, *}$, Chirag Paunwala ${ }^{1}$, Falgun Thakkar ${ }^{1}$, Heena Kher $^{1}$ and Mita Paunwala ${ }^{1}$ \\ ${ }^{1}$ Department of Electronics \& Communication Engineering, G. H. Patel College of Engineering \& Technology, Bakrol Road, Vallabh Vidyanagar- \\ 388120, Gujarat, India
}

Biomedical signals like ECG, EEG, EMG, EOG, ERG etc. and images such as ultrasound, MRI, CT, PET etc. are very much useful for assessing the wellbeing of a human being. In order to determine the abnormality in a particular organ or part of the body, physicians use these signals and images. Although, today's signal recorders and image scanners are of excellent resolution and quality, sometimes they fail to convey the actual scenario of the body part/ organ [1].

Soft computing and machine learning methods play important role in dealing with biomedical signals/ images and they have numerous applications like noise/ artifact removal from signals/ images, early detection of seizure/ cancer/ tumours, fusion of images for better diagnosis, classification of signals/ images and much more [2,3].

This special issue aims at compiling the novel research

\footnotetext{
* Address correspondence to this author at Department of Electronics \& Communication Engineering, G H Patel College of Engineering \& Technology, Bakrol Road, Vallabh Vidyanagar- 388120, Gujarat, India; Tel: +91 2692231651 (O); Fax: +91 2692 236896; E-mail: rahul2777@gmail.com
}

outcomes of various soft computing and machine learning algorithms applied to varieties of biomedical signals/ images. We have received an exceptional response to this thematic issue. Some of the notable contributions include Applications of Deep Learning and convolutional neural network for cancer detection, Content-based medical image retrieval, Tumour detection using MRI, COVID-19 screening using chest radiography, machine Learning based epileptic seizure detection [4] etc.

\section{REFERENCES}

[1] B. Suriya, and M. Raja, "Modelling Human Activity using Smartphone data", Open Biomed. J., vol. 15, 2021. [E-pub ahead of print].

[2] K. Dheeraj, M. A. Mayuri, and C. Indranath, "Empirical Analysis of Deep Convolutional Generative Adversarial Network for Ultrasound Image Synthesis", Open Biomed. J., vol. 15, 2019. [E-pub ahead of print].

[3] A. Rachel, L. Fiona, and R. Swarnalatha, "GSM and Arduino Based Vital Sign Monitoring System", Open Biomed. J., vol. 15, 2021. [Epub ahead of print].

[4] P. Vibha, T. Jaishree, and G. Amit, "Essentials of Predicting Epileptic Seizures Based on EEG Using Machine Learning: A Review", Open Biomed. J., vol. 15, 2021. [E-pub ahead of print].

This is an open access article distributed under the terms of the Creative Commons Attribution 4.0 International Public License (CC-BY 4.0), a copy of which is available at: $\mathrm{https}$ ://creativecommons.org/licenses/by/4.0/legalcode. This license permits unrestricted use, distribution, and reproduction in any medium, provided the original author and source are credited. 